\title{
LA TEORÍA DEL LENGUAJE EN LA FILOSOFÍA TRASCENDENTAL DE FITCHE
}

\author{
Luciano Corsico \\ Universidad Nacional de Rosario \\ Universidad Nacional de Lanús \\ (Argentina) \\ lucianocorsico@yahoo.com
}

\begin{abstract}
Resumen: En el presente artículo, mi principal objetivo es reconstruir brevemente la teoría fichteana del lenguaje, evaluando al mismo tiempo sus méritos y limitaciones dentro del panorama actual de la filosofía contemporánea. Es cierto que Fichte elabora una teoría del lenguaje, pero no le atribuye una función metodológica decisiva en el contexto de su sistema filosófico. El lenguaje es considerado como un objeto de la investigación filosófica, pero nunca como una condición trascendental del pensamiento, del conocimiento o de la acción. Sin embargo, Fichte posee el mérito de haber ofrecido una comprensión trascendental de la comunicación humana que aún tiene influencia en el debate filosófico contemporáneo.
\end{abstract}

Palabras clave: Lenguaje, Método, Idealismo, Conocimiento, Acción

\begin{abstract}
In this paper I will try to briefly reconstruct the Fichte's theory of language and then to evaluate also their merits and limitations within the field of contemporary philosophy. It is true that Fichte develops a theory of language, but he does not attribute to language an important methodological function in the context of his philosophical system. The language is considered as an object of philosophical research, but never as a transcendental condition of thinking, knowledge or action. However, we still have to consider the Fichte's transcendental explication of human communication as having an enormous influence on the contemporary philosophical debate.
\end{abstract}

Keywords: Language, Method, Idealism, Knowledge, Action 
Cuadernos Filosóficos. Segunda Época, XIII, 2016

\section{Introducción}

En las últimas décadas, la literatura especializada sobre la filosofía de Fichte ha destinado un lugar de enorme importancia a su teoría del lenguaje. No son pocas las interpretaciones y reconstrucciones del sistema fichteano de la Wissenschaftslere (WL) que se ocupan de su particular deducción del lenguaje como una verdadera anticipación del denominado giro lingüístico y de las actuales propuestas de la filosofía contemporánea. Según Jean Paul Surber, por ejemplo, la teoría fichteana sobre el lenguaje anticipa buena parte de las posiciones teóricas del post-estructuralismo sobre la relación entre la filosofía, la verdad y el fenómeno de la significación. Por ese motivo, la interpretación post-estructuralista del Idealismo Alemán debería ser revisada a partir de un estudio más adecuado sobre la concepción fichteana del lenguaje (Surber 1996, pp. 6-7). Thomas Sören Hoffmann también considera que la teoría del lenguaje cumple una función indispensable en la filosofía de Fichte. Por un lado, en su ensayo Von der Sprachfähigkeit und dem Ursprunge der Sprache de 1795, el lenguaje aparece como objeto de una construcción filosófica que se remonta hasta las formas gramaticales empíricas y particulares. Por otro lado, en Rede an die deutsche Nation de 1808, el lenguaje aparece como el medium en que se desarrolla la auto-construcción de la razón y adquiere el significado de una facultad originaria. En este último caso, teoría del lenguaje aparece como un tema exclusivo de la filosofía de Fichte (Hoffmann 1997, pp. 17-18). Por su parte, Axel Honneth considera que el célebre concepto fichteano de «exhortación» (Aufforderung) puede interpretarse legítimamente como una forma de acción dialógica y comunicativa. Según Honneth, Fichte piensa la exhortación como un «simple dirigir la palabra» (der bloßen Anrede) al otro (Honneth, 2001, pp. 74-77). Marek Siemek también comprende la exhortación fichteana como una acción esencialmente comunicativa. En su Grundlage des Naturrechts (GNR) de 1796/97, Fichte comprende la intersubjetividad como un principio clave para superar las aporías correspondientes a una explicación circular de la autoconciencia. Para evitar estas aporías, Fichte habría recurrido inteligentemente al carácter comunicativo de las relaciones interpersonales (Siemek, 2003, pp. 60$62)$.

A pesar de la tendencia impuesta por estas interpretaciones, resulta bastante difícil sostener la tesis de que Fichte anticipa las consecuencias del denominado giro lingüístico de la filosofía contemporánea. Si el análisis se limita a la base textual disponible, la filosofía de Fichte no parece ofrecer pruebas suficientes para sostener la existencia de una auténtica reformulación de los problemas filosóficos en términos lingüísticos. ${ }^{1}$ Es cierto que Fichte elabora una teoría del lenguaje,

\footnotetext{
${ }^{1}$ Las referencias a los textos de Fichte utilizados en el presente artículo corresponden siempre a la edición de sus obras completas contenidas en J. G. Fichte. Gesamtausgabe der Bayerischen Akademie der Wissenschaften, R. Lauth, H. Jacob., y Gliwitzky, H. (eds.), Frommann-Holzboog, Stuttgart/Bad Cannstatt, 1962, ss. En cada caso se utiliza la abreviatura GA, seguida por el número de volumen y la paginación correspondiente. A las citas del texto original en alemán que aparecen
} 
Cuadernos Filosóficos. Segunda Época, XIII, 2016

pero no le atribuye una función metodológica decisiva en el contexto de su sistema filosófico. Como sucede con otros pensadores de la modernidad, el lenguaje es considerado más bien como un objeto de la investigación filosófica, pero nunca como una condición trascendental del pensamiento, del conocimiento o de la acción. Ahora bien, aunque la concepción fichteana del lenguaje sea limitada y no pueda compararse con los actuales enfoques filosóficos, su deducción del lenguaje tiene el mérito de incorporar una dimensión trascendental al debate sobre la esencia de la comunicación humana. Esta perspectiva trascendental no corresponde a una visión anacrónica de la filosofía que se agotaría en el siglo XIX, sino que tiene una evidente continuidad en la filosofía contemporánea (por ejemplo, en el proyecto de la pragmática trascendental contemporánea de Karl-Otto Apel y Wolfgang Kuhlmann).

En el presente artículo, mi principal objetivo es reconstruir brevemente la teoría fichteana del lenguaje, sin dejar de evaluar sus méritos y limitaciones dentro del panorama actual de la filosofía contemporánea. Para alcanzar una mayor claridad en el desarrollo de mi análisis, divido este artículo en tres secciones. En la primera sección (I), intento reconstruir la concepción de Fichte sobre el lenguaje durante el período de Jena (1794-1799). En la segunda sección (II), es analizada la concepción fichteana del lenguaje durante el período de Berlin (1800-1814). En la tercera sección (III), intento ofrecer una reconstrucción del concepto fichteano de exhortación (Aufforderung), para determinar si efectivamente puede ser interpretado como una interacción comunicativa entre seres racionales. Finalmente, extraigo algunas conclusiones sobre el tema analizado en el presente trabajo. En verdad, Fichte rechaza de manera explícita la determinación del lenguaje sobre el pensamiento durante el período de Jena. Durante ese período, Fichte se resiste a caracterizar explícitamente la exhortación como un acto de habla e incluso admite que el reconocimiento entre seres racionales no es necesariamente articulado en el lenguaje verbal, sino que puede fundamentarse en la mera contemplación de la figura humana en estado de reposo. Durante el período de Berlín, donde parece invertirse el orden de la relación entre lenguaje y pensamiento, Fichte tampoco desarrolla una reflexión explícita sobre las condiciones linguiísticas del pensamiento y de la acción. En resumen, la filosofía de Fichte tiene el mérito de ofrecer una concepción trascendental del lenguaje humano, pero no anticipa el giro lingüístico de la filosofía contemporánea, sino que se mantiene en todo momento dentro del paradigma de una filosofía moderna de la conciencia. No obstante, su deducción trascendental tuvo del mérito de ofrecer una original perspectiva sobre el problema de la comunicación humana, evitando las consecuencias relativistas de una concepción meramente empírica del lenguaje.

en el cuerpo del texto, siempre se agrega una traducción al castellano en una nota a pie de página. Las traducciones pertenecen en todos los casos al autor del presente artículo. 


\section{La concepción fichteana del lenguaje durante el período de Jena (1794- 1799)}

Durante su estadía en la Universidad de Jena (1794-1799), Fichte elabora la primera versión de su sistema de filosofía denominado «Doctrina de la Ciencia»(Wissenschaftslehre). A grandes rasgos, puede decirse que ese sistema contenía una metafísica o philosophia prima, una teoría ética y una teoría del derecho. Durante ese período, sus escritos sobre religión no alcanzan nunca el mismo nivel de desarrollo teórico y sistemático, sino que se mantienen más bien en un registro meramente popular. La doctrina fichteana de la religión se incorpora a su sistema de filosofía en un período posterior. Durante el período de Jena, Fichte fundamenta todo su sistema en el principio de un Yo absoluto, que tiene el carácter de una exigencia práctica y moral. A este principio de un Yo absoluto, se opone siempre el principio de un «No-Yo» (Nicht-Ich), que es un término utilizado por Fichte para referirse a toda aquella realidad que se opone al sujeto. Este No-Yo abarca al mismo tiempo la realidad de la naturaleza y la realidad del entorno social humano. A partir de un método reflexivo, Fichte analiza la permanente interacción entre el Yo y el No-Yo, dentro de los distintas esferas de la actividad humana. Con el auxilio de este método reflexivo, que fue inspirado por la filosofía trascendental kantiana, Fichte desarrolla una deducción sistemática de los principios fundametales del saber humano, la ética y el derecho. En la construcción sistemática de su Wissenschaftslehre (WL), Fichte intenta ofrecer una fundamentación última de la filosofía trascendental y un desarrollo exhaustivo del primado kantiano de la razón práctica.

Durante este primer período de su producción filosófica, aparece también una temprana reflexión de Fichte sobre los principios trascendentales del lenguaje humano. En efecto, una exposición ordenada del problema del lenguaje aparece por primera vez en su ensayo Von der Sprachfähigkeit und dem Ursprunge der Sprache del año 1795. Desde luego, este ensayo de Fichte puede ubicarse en el contexto de algunas discusiones filosóficas previas sobre el origen y la importancia del lenguaje. A ese mismo contexto pertenecen, por ejemplo, las teorías sobre el origen del lenguaje de autores como Süßmilch, Rousseau o Condillac. No obstante, el ensayo de Fichte parece tener una relación más directa con los escritos de Herder y J. G. Hamann. La concepción fichteana del lenguaje surge precisamente en el marco de un debate con estos pensadores de su época.

En su Abhandlung über den Ursprung der Sprache (1772), por ejemplo, Herder se había ocupado de discutir la tesis de un origen "divino” del lenguaje. Según la posición de Herder, el origen de la capacidad lingüística está relacionada con la constitución del organismo humano, así como el 
Cuadernos Filosóficos. Segunda Época, XIII, 2016

desarrollo del lenguaje está vinculado con una teleología de la naturaleza. Por un lado, Herder considera que un hombre puede designar un objeto o un pensamiento a través del lenguaje, en la medida en que posee cierta capacidad de reflexión. Herder entiende la reflexión como una capacidad que está presente en toda actividad corporal consciente de un ser humano. El hombre posee esta capacidad de reflexionar como una especie de dotación natural, similar al instinto en los animales. Por otro lado, Herder considera que la naturaleza no ha otorgado al hombre esta facultad en vano. A través de la reflexión y del consecuente desarrollo del lenguaje, el hombre no sólo puede preservar su especie, sino también orientarse hacia un constante progreso (Dahlstrom, 2005: 84-85).

El ensayo de Fichte sobre el lenguaje puede comprenderse también como una respuesta directa a las objeciones formuladas por J. G. Hamann contra la filosofía kantiana. En el año 1784, Hamann había redactado un escrito titulado Metakritik über den Purismum der Vernunft, dirigido contra el proyecto de la filosofía crítica de Kant. Aunque la Metakritik de Hamann no fue publicada hasta el año 1800, el contenido de sus manuscritos comenzó a circular mucho tiempo antes en el ambiente intelectual de la época, sobre todo gracias a la difusión de Herder y Jacobi. La publicación del escrito de Hamann parece haber sido forzada por un enemigo de Herder. En efecto, Herder había publicado en 1799 un escrito titulado Verstand und Erfahrung. Eine Metakritik zur Kritik der reinen Vernunft y un enemigo suyo pretendía demostrar su supuesto plagio (Dahlstrom, 2005: 78). En cualquier caso, lo cierto es que la Metakritik de Hamann acusaba a Kant de efectuar una hipóstasis de la razón. La filosofía de Kant aislaba a la razón de su existencia concreta en la experiencia, en la historia y especialmente en el lenguaje. Según Hamann, el uso de la razón presupone el lenguaje como su condición previa. Por ese motivo, el proyecto de una crítica de la razón propuesto por Kant requiere asimismo de una previa metacrítica del lenguaje.

Fichte estaba familiarizado con las ideas de Hamann y comprendió inmediatamente que su metacrítica lingüística (en principio dirigida contra la filosofía de Kant) representaba una amenaza para cualquier teoría que pretendiera establecer las necesarias estructuras a priori de la razón a partir de un punto de vista trascendental y a-histórico (incluyendo, por supuesto, su propia WL). Su ensayo sobre el origen del lenguaje puede entenderse entonces como una respuesta a este proyecto de una metacrítica de la razón pura. De manera consecuente, Fichte tenía que aplicar al lenguaje el mismo método de deducción trascendental, que ya había aplicado en la elaboración de su WL. De este modo, Fichte no sólo podía responder a las objeciones de Hamann. También podía demostrar la superioridad de su propia versión de la filosofía trascendental frente a la versión anterior de Kant, que no había podido responder a esas objeciones (Surber, 1996: 11-12).

En su ensayo Von der Sprachfähigkeit und dem Ursprunge der Sprache de 1795, Fichte define el lenguaje como la expresión de nuestro pensamiento a través de signos arbitrarios (Fichte, GA I/3, 
Cuadernos Filosóficos. Segunda Época, XIII, 2016

97). En este contexto, Fichte parece emplear el término «arbitrario» (willkürlich) con al menos dos significados diferentes. Por un lado, Fichte nos dice que el signo lingüístico es arbitrario, en el sentido de que es utilizado por el hablante de manera intencional o según su propio arbitrio. Según Fichte, la utilización de símbolos o signos de manera explícita (ausdrücklich) es un requisito indispensable para definir el fenómeno del lenguaje humano. Es cierto, explica Fichte, que el pensamiento de un sujeto puede inferirse también a partir de sus acciones y de los efectos que produce en el mundo sensible. Mi acción es una consecuencia directa de mi pensamiento y, por este motivo, otro ser racional puede inferir ese pensamiento a partir de las acciones que realizo en el mundo fenoménico. Podría decirse entonces que toda actividad humana es lingüística, en la medida en que expresa un pensamiento. Sin embargo, Fichte niega esta última posibilidad. En el lenguaje, la utilización de signos por parte del sujeto debe ser intencional y explícita (Fichte, GA I/3, 98).

En otras palabras, Fichte considera que, en el desarrollo de la actividad lingüística, la principal finalidad del sujeto debe consistir en expresar a otros su pensamiento. En ese sentido, la voluntad tiene que determinarse de un modo específico para la realización de esa tarea. En las restantes acciones, no hay una utilización consciente y voluntaria de signos orientada hacia el fin de la comunicación. Por ejemplo, cuando alguien come, no posee necesariamente la intención de comunicar a otros que tiene hambre, aun cuando los demás puedan inferirlo a partir de la observación de su comportamiento. En consecuencia, Fichte considera que la expresión involuntaria de pensamientos o de sentimientos no es un lenguaje. La actividad lingüística presupone una voluntad determinada hacia el fin específico de la comunicación. Según Fichte, un uso involuntario (unwillkürlicher Gebrauch) del lenguaje implicaría una contradicción interna (Fichte, GA I/3, 98).

Pero, además, Fichte sostiene que el signo lingüístico es «arbitrario» en el sentido de que puede cumplir su función representativa, sin mantener ninguna relación de analogía con el objeto representado. Es decir, la relación entre el significante y el significado no está condicionada por una especie de isomorfismo entre ambos elementos del lenguaje, sino que es completamente convencional y arbitraria. La presunta semejanza natural entre el signo y el objeto designado carece de importancia. Dice Fichte:

«Ich habe mich bei der Erklärung der Sprache des Ausdrucks «wirkürliche Zeichen» bedient. Darunter verstehe ich hier solche Zeichen, welche ausdrücklich dazu bestimmt sind, diesen oder jenen Begriff anzudeuten. Ob dieselben mit dem Bezeichneten natüliche Aehnlichkeit haben, oder nicht, das ist hier völlig gleichgültig. Ich mag zu dem andern das Wort Fisch sagen - ein Zeichen, das mit dem Gegenstande, welchen es ausdrücken soll, gar keine Aehnlichkeit hat - oder ich mag ihm einen Fisch vorzeichnen; ein Zeichen, das mit dem 
Cuadernos Filosóficos. Segunda Época, XIII, 2016

Bezeichneten allerdings Aehnlichkeit hat - in beiden Fällen habe ich keinen Zweck, als den, die Vorstellung eines bestimmten Gegenstandes bei dem andern zu veranlassen- folglich kommen beide Zeichen darinn überein, daß sie willkürlich sind» (Fichte, GA I/3, 98). ${ }^{2}$

En resumen, puede decirse que Fichte considera que el uso intencional de signos arbitrarios destinados a expresar el contenido del pensamiento constituye el principal criterio para distinguir entre una actividad lingüística y una actividad no-lingüística. El uso del lenguaje no puede ser involuntario, implícito o casual. En la acción comunicativa, la voluntad de expresar el propio pensamiento debe ser consciente y explícita por parte del sujeto. La relación de semejanza con el objeto del pensamiento o de la representación resulta indiferente. Ciertamente, el acto de pensamiento expresado luego por el lenguaje puede estar implícito en una acción, pero la actividad lingüística de comunicar ese pensamiento a otros tiene que ser siempre explícita. De este modo, Fichte traza una nítida distinción entre lenguaje y pensamiento. El «lenguaje» (Sprache) es solamente la tarea específica de comunicar a otros el pensamiento por medio de signos audibles o visibles. En cambio, el pensamiento es una actividad intelectual que puede desarrollarse sin necesidad de recurrir a signos lingüísticos. Según Fichte, algunos intelectuales de su época le han concedido una importancia excesiva al lenguaje, como si no fuera posible pensar sin el auxilio de palabras o de signos. Dice Fichte:

«Ich beweise hier nicht, daß der Mensch ohne Sprache nicht denken, und ohne sie keine allgemeinen abstracten Begriffe haben könne. Das kann er allerdings vermittelst der Bilder, die er durch die Phantasie sich entwirft. Die Sprache ist meiner Ueberzeugung nach für viel zu wichtig gehalten worden, wenn man geglaubt hat, daß ohne sie überhaupt kein Vernunftgebrauch statt gefunden haben würde» (Fichte, GA I/3, 103). ${ }^{3}$

En este punto, Fichte manifiesta una clara oposición frente a la perspectiva adoptada por Hamann en su Metakritik über den Purismum der Vernunft. Fichte evita subordinar la razón al uso del lenguaje. Aunque Fichte admite que el lenguaje es un instrumento indispensable para expresar el

\footnotetext{
2 «En la explicación del lenguaje, me he servido de la expresión «signos arbitrarios». Bajo esta expresión, entiendo aquí aquellos signos que están expresamente determinados a indicar este o aquel concepto. Resulta aquí completamente indistinto si estos signos tienen o no una semejanza natural con el objeto designado. Yo puedo decirle a otro la palabra «pez» - un signo que no tiene ninguna semejanza con el objeto que debe expresar- o puedo dibujarle a él un pez - un signo que en efecto tiene una semejanza con el objeto designado. En ambos casos, yo no tengo otro fin que ocasionar en el otro la representación de un determinado objeto. Por consiguiente, ambos signos concuerdan en el hecho de que son arbitrarios».

3 «Yo no demuestro aquí que el hombre no pueda pensar sin lenguaje y que no pueda tener conceptos generales abstractos sin lenguaje. Eso puede hacerlo efectivamente por medio de las imágenes que se elaboran a través de la fantasía. Según mi convicción, el lenguaje ha llegado a ser tan importante para muchos, cuando se ha creido que, sin lenguaje, no podría haber tenido lugar el uso de la razón en general».
} 
Cuadernos Filosóficos. Segunda Época, XIII, 2016

pensamiento y para reconocer a otros como seres racionales, se resiste a afirmar una primacía de la capacidad lingüística sobre la razón, así como también niega una posible identificación entre ambas. Desde la perspectiva de Fichte, alguien puede pensar y comportarse como un ser racional, aun cuando no ejerza su facultad lingüística (Surber, 1996: 37).

Por otra parte, es cierto que Fichte no intenta ofrecer una explicación empírica, sino una deducción trascendental del lenguaje. A pesar del carácter arbitrario adjudicado a los signos particulares, Fichte no considera el lenguaje en general como un producto casual derivado de ciertas convenciones o acuerdos, sino más bien como una herramienta de comunicación cuyo origen se puede justificar necesariamente a partir de la propia estructura de la conciencia humana. Según Fichte, la invención del lenguaje no es un hecho contingente de la historia, sino que ha estado siempre sometida a leyes necesarias de la razón. En el contexto de esta deducción del lenguaje, Fichte se refiere implícitamente a los principios fundamentales del saber humano establecidos en su Grundlage der gesammten Wissenschaftslehre (GWL) de 1794/95. Aunque utiliza en este contexto una terminología distinta, aparentemente extraída de la ciencia natural y de estudios de carácter antropológico, puede decirse que Fichte pretende ofrecer una auténtica deducción trascendental del lenguaje. A través de esa terminología que se refiere constantemente a la oposición entre el hombre y la naturaleza, o entre el individuo y la sociedad, Fichte sugiere constantemente la tesis de que el lenguaje se origina a partir de la necesaria interacción entre Yo y No-Yo. ${ }^{4}$

En ese sentido debe interpretarse la argumentación de Fichte, que se refiere a las dos maneras alternativas que tiene el hombre de relacionarse con el mundo en general. En una terminología específicamente fichteana, podría decirse que el Yo individual tiene dos maneras alternativas de comportarse frente al No-Yo. Cuando el No-Yo es comprendido como naturaleza, el Yo pretende modificarlo y someterlo completamente (Fichte, GA I/3, 100). En otras palabras, el sujeto intenta transformar la naturaleza no-racional, con el fin de someterla a sus propios principios racionales. Sólo

\footnotetext{
${ }^{4}$ Como ya se señaló más arriba, Fichte utiliza el singular concepto de No-Yo (Nicht-Ich) para designar todo aquello que se opone a un Yo: el mundo natural y el mundo social e histórico. Este principio cumple una doble función en el célebre escrito titulado Grundlage der gesammten Wissenschaftslehre de 1794/95, donde Fichte ofrece una fundamentación de todo su sistema. En primer lugar, el principio del No-Yo cumple una función decisiva en la representación, como una instancia determinante para la fundamentación del saber teórico. Según Fichte, el principio del saber teórico puede formularse de la siguiente manera: el Yo se pone a sí mismo como determinado por el No-Yo (GA I/2, 287). La representación de objetos es la actividad teórica por antonomasia y puede concebirse precisamente como una actividad en la cual el propio sujeto se encuentra determinado por la realidad que intenta conocer y representar. Por ese motivo, cuando intenta conocer el mundo, el sujeto está obligado a pensarse a sí mismo como un Yo que se encuentra sometido a la determinación del No-Yo. En segundo lugar, el principio del No-Yo cumple una función muy significativa también en el ámbito del saber práctico. Según Fichte, el principio del saber práctico puede formularse de la siguiente manera: el Yo se pone a sí mismo como determinando al No-Yo (GA I/2, 385). Desde la perspectiva de la praxis, el sujeto siempre se piensa a sí mismo como un sujeto que se auto-determina y que puede luego determinar la realidad opuesta a él (en terminología fichteana, el No-Yo). En otras palabras, el Yo debe intentar someter siempre la realidad exterior a sus propios principios racionales a través de la praxis. En la acción, el sujeto se piensa siempre como un Yo que debe determinar al No-Yo.
} 
Cuadernos Filosóficos. Segunda Época, XIII, 2016

bajo esta condición, es posible para el sujeto cumplir con la máxima exigencia de la razón práctica: el Yo tiene que ponerse absolutamente a sí mismo. Cuando el sujeto se mantiene simplemente en una actitud contemplativa, representando o describiendo las cosas tal como son, se produce un desacuerdo con sus propias exigencias racionales. El sujeto tiene que modificar la realidad natural a través de la praxis, para que finalmente concuerde con su ideal y de esta manera imponer por la fuerza las leyes de la razón a la propia naturaleza (Fichte, GA I/3, 100-101). Pero cuando el Yo se opone al No-Yo como mundo social y humano tiene que actuar de una manera diferente. En el mundo social, el hombre no se opone a una naturaleza bruta, sino a otros hombres que comparten con él los mismos principios de racionalidad. El reconocimiento de esa racionalidad requiere la posibilidad de una interacción o un intercambio entre sujetos, de la cual resulta una correspondiente modificación de la acción con respecto a fines.

No puedo reconocer como racional a un ser que actúa invariablemente o de forma mecánica, independientemente de cualquier interacción que yo pueda tener con él. Según Fichte, sólo puedo reconocer como racional a un ser que modifica sus intenciones, después de entender un acto de comunicación en el que yo expreso mis propias intenciones (Fichte, GA I/3, 101). Por ese motivo, la posibilidad de reconocer la racionalidad fuera de la esfera subjetiva necesita la invención del lenguaje. Es decir, el reconocimiento mutuo de la racionalidad entre seres de la especie humana depende de la invención de un medio de comunicación adecuado de los pensamientos e intenciones. Dice Fichte:

«So wie es mir mit andern geht, so andern mit mir. Wie leicht kann ich die wohlmeinende Handlung eines andern misverstehen, und mit Undank vergelten? So wie ich aber seine Absicht besser einsehe, so werde ich wunschen mein Vergehen wieder gut zu machen, und um deswillen von seinen Gedanken kunftig besser unterrichtet zu sein. - Ich wunsche also, das der andere meine Absicht wissen moge, damit er mir nicht zuwider handle, und aus gleichem Grunde wunsche ich, die Absichten des andern zu wissen. Daher die Aufgabe zur Erfindung gewisser Zeichen, wodurch wir andern unsere Gedanken mittheilen konnen» (Fichte, GA I/3, 102). ${ }^{5}$

Sin lenguaje, se vuelve imposible la vida social, porque las acciones de los hombres siempre son susceptibles de una interpretación incorrecta. Los signos del lenguaje están elaborados voluntariamente por el hombre para resolver este problema. Sólo por medio de la utilización de signos lingüísticos puedo comunicar (con el menor margen de ambigüedad posible) mis propias intenciones

\footnotetext{
5 «Así como yo me comporto con el otro, el otro se comporta conmigo. ¡Con qué facilidad puedo malinterpretar la acción bien intencionada del otro y retribuirla con ingratitud! Pero cuando comprendo mejor su intención, entonces yo deseo remediar mi falta y estar mejor informado en el futuro sobre sus pensamientos. Yo deseo así que el otro pueda conocer mi intención, para que no me trate con desprecio, y por iguales motivos deseo conocer las intenciones del otro. Por eso, surge la tarea de inventar ciertos signos, por medio de los cuales podemos comunicar a otros nuestros pensamientos».
} 
Cuadernos Filosóficos. Segunda Época, XIII, 2016

y conocer las intenciones de los demás. De esta manera, surge la idea de un lenguaje en el contexto de la interacción social humana. El lenguaje se fundamenta igualmente en la tendencia a convertir todo No-Yo en un Yo y en la tendencia a extender las leyes de la racionalidad fuera de los límites de la conciencia subjetiva (Fichte, GA I/3, 102-103).

\section{La concepción fichteana del lenguaje durante el período de Berlín (1800-}

\section{4)}

A pesar de este esfuerzo de deducción trascendental, debe admitirse que Fichte no atribuye demasiada importancia al lenguaje en el marco de su exposición sistemática de la WL durante el período de Jena (1794-1799) y sólo lo considera como un tema secundario en la investigación filosófica. Esta comprensión del lenguaje por parte de Fichte no se modifica substancialmente en sus escritos posteriores del período de Berlín (1800-1814). Aún cuando su Rede an die deutsche Nation (1808) parece ofrecer nuevas consideraciones sobre la facultad lingüística del hombre, su teoría fundamental se mantiene intacta. En cierto sentido, Fichte intenta conservar la misma comprensión trascendental del lenguaje que ya había elaborado previamente en su escrito de 1795. De manera consecuente, Fichte vuelve a afirmar que el lenguaje expresa una legalidad superior que no puede identificarse meramente con las convenciones idiomáticas. Dice Fichte:

«Die Sprache uberhaupt, und besonders die Bezeichnung der Gegenstande in derselben durch das Lautwerden der Sprachwerkzeuge hangt keinesweges von willkührlichen Beschlussen, und Verabredungen ab, sondern es giebt zuvorderst ein Grundgesez, nach welchem jedweder Begriff in den menschlichen Sprachwerkzeugen zu diesem, und keinem andern Laute wird. So wie die Gegenstande sich in den Sinnenwerkzeugen des Einzelnen mit dieser bestimmten Figur, Farbe, u. s.w. abbilden, so bilden sie sich im Werkzeuge des gesellschaftlichen Menschen, in der Sprache, mit diesem bestimmten Laute ab. Nicht eigentlich redet der Mensch, sondern in ihm redet die menschliche Natur, und verkundiget sich andern seines Gleichen. Und so muste man sagen: die Sprache ist eine einzige, und durchaus nothwendige» (Fichte, GA I/10, 146). ${ }^{6}$

Como puede observarse en el pasaje citado, Fichte ofrece una versión aún más radical de su concepción trascendental del lenguaje humano e incluso intenta reducir a su mínima expresión el carácter arbitrario del signo lingüístico. Los diversos sonidos y las distintas variaciones idiomáticas

\footnotetext{
6 «El lenguaje en general, y en particular la designación de objetos en el mismo por medio de los sonidos del instrumento del habla no depende en modo alguno de convenios y de acuerdos arbitrarios, sino que existe primordialmente una ley fundamental, según la cual todo concepto en el instrumento del habla humana se convierte en este sonido y en ningún otro. Así como los objetos se reproducen en el instrumento de los sentidos del individuo con esta determinada figura, color, etc., así se reproducen en el lenguaje, que es el instrumento del hombre en sociedad, con determinados sonidos. No habla realmente el hombre, sino que en él habla la naturaleza humana, y se anuncia a los otros semejantes a él. Y así tendría que decirse: el lenguaje es único y absolutamente necesario».
} 
Cuadernos Filosóficos. Segunda Época, XIII, 2016

se fundamentan en un lenguaje puro. Este lenguaje contiene una ley inherente que permanece constante a través de todos los cambios y modificaciones que surgen durante su desarrollo histórico. El lenguaje es el mismo en todos los individuos que se encuentran en comunicación permanente. Según Fichte, la unidad de ese lenguaje originario permite explicar la continuidad de una lengua a través de sus diversas modificaciones temporales e históricas. Aunque después de algún tiempo puede ocurrir que los descendientes de un pueblo no comprendan la lengua de sus antepasados, la evolución permanente y sin interrupciones de la lengua nunca impide que los contemporáneos puedan entenderse entre sí. Según Fichte, todo pueblo tiene un eterno mediador e intérprete a través de la historia: el lenguaje puro que comparten todos sus miembros (Fichte, GA I/10, 146-147).

Como puede verse, Fichte pretende conservar una comprensión trascendental del lenguaje en su Rede an die deutsche Nation. Sin embargo, ese escrito incorpora además una nueva perspectiva sobre el tema. En efecto, Fichte no se limita a reflexionar sobre un concepto abstracto del lenguaje, sino que establece de manera especulativa una conexión directa entre el lenguaje y la vida espiritual de los pueblos. Dice Fichte:

«So verhalt es sich, sage ich, mit einer Sprache, die von dem ersten Laute an, der in derselben Volke ausbrach, ununterbrochen aus dem wirklichen gemeinsamen Leben dieses Volks sich entwickelt hat, und in die niemals ein Bestandtheil gekommen, der nicht eine wirklich erlebte Anschauung dieses Volks, und eine mit allen ubrigen Anschauungen desselben Volks im allseitig eingreifenden Zusammenhange stehende Anschauung ausdruckte» (Fichte, GA I/10, 150).?

El lenguaje aparece entonces como una fuerza concreta y supra-individual que influye de manera directa en la vida de los hombres. Esto sucede precisamente con la lengua del pueblo alemán, que se ha desarrollado sin interrupciones desde el origen de su vida espiritual. En esa lengua no hay elementos extraños: todos sus elementos constitutivos expresan una idea realmente experimentada por ese pueblo en conexión con la totalidad de su desarrollo. Si se incorporasen a ese pueblo individuos extraños, incapaces de comprender las ideas del pueblo originario expresadas en la lengua, permanecerían mudos dentro de la comunidad. Tampoco tendrían ninguna posibilidad de ejercer un influjo sobre la lengua, hasta que no se incorporasen al círculo (Umkreis) de las intuiciones compartidas por ese pueblo. Esta limitación que experimenta quien se incorpora a una comunidad extraña permite inferir precisamente que no son los individuos los que configuran el lenguaje, sino que más bien el lenguaje los configura a ellos (Fichte, GA I/10, 150).

\footnotetext{
${ }^{7}$ «Así se comporta un lenguaje, digo yo, que se ha desarrollado, desde la aparición del primer sonido en el mismo pueblo, de manera ininterrumpida a partir de la vida verdaderamente en común de este pueblo, en la que nunca surgió un elemento constitutivo que no expresara una intuición de este pueblo realmente experimentada ni una intuición que no se encontrara por todas partes en una conexión de interacción con todas las demás intuiciones de este mismo pueblo».
} 
En este nuevo contexto, Fichte sigue atribuyendo al lenguaje la función de expresar el pensamiento o de designar un concepto. Sin embargo, el concepto ya no se considera como un producto de la actividad individual del sujeto, sino como el resultado de la vida colectiva de un pueblo. Toda la historia de un pueblo puede reconstruirse entonces a partir de un concepto y su expresión simbólica en el lenguaje. Pero, además, Fichte atribuye al lenguaje una influencia decisiva sobre los pensamientos y las acciones humanas. Según Fichte, el lenguaje acompaña al individuo en su pensamiento y en su voluntad, estimulando su actividad o poniéndole límites muy precisos. Dice Fichte:

«Welchen unermeslichen Einflus auf die ganze menschliche Entwicklung eines Volks die Beschaffenheit seiner Sprache haben moge, der Sprache, welche den Einzelnen bis in die geheimste Tiefe seines Gemuths bei Denken, und Wollen begleitet, und beschrankt oder beflugelt, welche die gesammte Menschenmenge, die dieselbe redet, auf ihrem Gebiete zu einem einzigen gemeinsamen Verstande verknupft, welche der wahre gegenseitige Durchstromungspunkt der Sinnenwelt, und der Geister ist, und die Enden dieser beiden also in einander verschmilzt, das gar nicht zu sagen ist, zu welcher von beiden sie selber gehöre; wie verschieden die Folge dieses Einflusses ausfallen moge, da, wo das Verhaltnis ist, wie Leben, und Tod, last sich imAllgemeinen errathen»(GA I/10, 155). ${ }^{8}$

En cierto sentido, podría decirse que Fichte invierte aquí la relación entre pensamiento y lenguaje que generalmente puede observarse en el contexto de una filosofía de la conciencia. Como el propio Fichte parece reconocer en el pasaje citado, el pensamiento subjetivo se encuentra determinado por el lenguaje y no a la inversa. Sin embargo, no resultaría legítimo sostener que la WL anticipa de este modo los posteriores resultados del giro lingüístico de la filosofía contemporánea. En realidad, Fichte nunca propone un análisis filosófico de los componentes sintácticos, semánticos o pragmáticos de la argumentación o de los actos de habla en general. Incluso la función metodológica del lenguaje en el contexto de la investigación filosófica resulta ignorada por completo en el ámbito sistemático de su WL.

En lugar de incorporar sus reflexiones sobre el lenguaje a la formulación explícita de un criterio metodológico (como sucede en las propuestas de la filosofía contemporánea), Fichte las

\footnotetext{
8 «Puede adivinarse en general cuál es el inconmensurable influjo que puede tener la naturaleza de un lenguaje sobre la totalidad del desarrollo humano de un pueblo, de un lenguaje, que acompaña al individuo en el pensamiento y en la voluntad hasta la profundidad más secreta de su alma, y lo limita o estimula; un lenguaje, que relaciona a toda la multitud de hombres que hablan esta lengua en un mismo territorio con un único entendimiento general; un lenguaje que es el verdadero punto de atravesamiento recíproco del mundo sensible y del mundo espiritual, y que así fusiona los confines de ambos uno en el otro, de manera que no pueda decirse en absoluto a cuál de los dos pertenece el lenguaje; y también puede adivinarse en general cuáles son las diversas consecuencias que pueden resultar de este influjo allí donde la relación es como la vida y la muerte».
} 
Cuadernos Filosóficos. Segunda Época, XIII, 2016

incluye en su doctrina sobre la espiritualidad viviente de Dios. Según Fichte, la lengua viviente de un pueblo refleja la actividad infinita de Dios, porque permite el continuo perfeccionamiento de las condiciones humanas según la imagen divina. La actividad eterna de Dios nunca se completa, sino que continúa hasta el infinito. De forma paralela, un lenguaje viviente (lebendigen Sprache) permite estimular la actividad infinita del pensamiento por parte de todos los miembros de una comunidad. En la permanente transformación de una lengua viva, las palabras y los significados se amplían constantemente. Por ese motivo, el vínculo de un pueblo con Dios, que es la fuente de toda vida espiritual, sólo es posible a través del desarrollo de ese lenguaje viviente (Fichte, GA I/10, 157-158).

Como puede observarse, las nuevas consideraciones que Fichte introduce en sus escritos del período de Berlín no contribuyen a modificar substancialmente su primera interpretación sobre el fenómeno del lenguaje humano. Fichte no deja de concebir el lenguaje como un posible objeto de conocimiento entre otros y nunca lo incorpora a la WL como una condición indispensable de la propia reflexión filosófica. En otras palabras, Fichte nunca desarrolla una teoría explícita sobre los presupuestos lingüísticos del saber filosófico. En la exposición sistemática de su WL, el lenguaje se convierte en objeto de una deducción trascendental, pero nunca en una condición de posibilidad del propio procedimiento deductivo.

\section{La exhortación como acción comunicativa}

Antes de finalizar, quisiera ocuparme de un concepto que Fichte utiliza en el marco de su pensamiento jurídico-político, pero que tiene una estrecha conexión con su teoría del lenguaje. Me refiero más precisamente al concepto de «exhortación» (Aufforderung), que Fichte expone y desarrolla en su Grundlage des Naturrechts (GNR) de 1796/97. Según Fichte, este concepto de exhortación designa una influencia externa sobre el sujeto, pero que no suprime su libertad o su capacidad de autodeterminación. En virtud de una exhortación, se efectúa sólo una determinación del sujeto a la autodeterminación (Fichte, GA I/3, 342). Cuando el sujeto recibe una exhortación a actuar libremente, puede cumplirla de dos maneras distintas. Por un lado, el sujeto puede cumplir con la exhortación a través de un actuar efectivo. En tanto actúa, el sujeto decide actuar de un modo determinado. En la esfera de sus posibles acciones, el sujeto es exhortado a elegir una acción determinada a través de una absoluta autodeterminación. De este modo, el sujeto que realiza una acción determinada conserva su carácter de ser racional y libre, pero cumple también con la exhortación recibida. Por otro lado, Fichte señala también que el sujeto puede cumplir con esa misma exhortación a través de un no-actuar (durch Nichthandeln). El sujeto conserva su racionalidad y su libertad también cuando decide no realizar ninguna acción, porque esa decisión presupone la 
Cuadernos Filosóficos. Segunda Época, XIII, 2016

comprensión de su propia actividad como algo exigido de él. Aunque el sujeto pueda proceder contra la expectativa de actuar de manera efectiva, cumple sin embargo con la exigencia de autodeterminarse libremente. Cuando se abstiene de realizar una determinada acción, el sujeto también decide con total libertad entre actuar y no actuar (Fichte, GA I/3, 343).

Fichte muestra así que la actividad de la exhortación presupone la interacción entre dos seres racionales: el sujeto que recibe la exhortación y el sujeto que la efectúa. El objetivo final de la exhortación es demandar al otro la realización de una acción libre y autodeterminada. Por ese motivo, explica Fichte, el ser racional que recibe una exhortación no puede considerarse nunca como un ser forzado a actuar de una determinada manera. La exhortación tiene el carácter de una demanda que el otro puede satisfacer o no. En este aspecto, la influencia que la exhortación ejerce sobre el ser racional no puede compararse con una determinación causal que un objeto material puede producir sobre otro de la misma índole. La relación entre dos seres racionales a través de la exhortación no puede identificarse con la relación causa-efecto entre dos cuerpos físicos. Estos cuerpos pueden actuar unos sobre otros, sin necesidad de comprender su propia actividad. La exhortación, en cambio, requiere la comprensión del significado de ciertos conceptos, que se encuentran expresados en la exigencia dirigida a otro ser racional.

La mencionada exhortación no demanda cualquier tipo de acción, sino una acción libre y autodeterminada. La exhortación no representa una simple influencia sobre un sujeto, sino una influencia que no suprime su libertad o su genuina racionalidad. Para que la exhortación pueda resultar efectiva y cumplir con su finalidad, debe dirigirse a un ser racional, es decir, a un ser que puede comprender el significado de esa exhortación. Ahora bien, para comprender el significado de la exhortación, el ser racional que recibe esa exhortación debe poseer los conceptos de razón y de libertad. Pero esta comprensión del significado de la exhortación debe ser recíproca: no sólo debe comprenderla quien la recibe, sino también quien la efectúa. Quien produce una exhortación no puede ser un cuerpo material e inerte, sino una inteligencia (eine Intelligenz). En otras palabras, quien es capaz de dirigir a otro una exhortación debe también comprenderse como un ser racional (Fichte, GA $\mathrm{I} / 3,345)$.

Según Fichte, el concepto de exhortación es el más adecuado para caracterizar la interacción entre seres racionales y libres. Como una actividad fundada en el concepto, la exhortación es una actividad dirigida al otro como un ser racional. En tanto propone la libre ejecución de una acción, sin determinarla a través del uso de la fuerza, el recurso a la exhortación por parte de un ser racional garantiza la libertad de otro ser racional opuesto a él. En este sentido, la exhortación es una actividad libre, pero que, al mismo tiempo, no excluye la libertad de los demás. Si un ser racional desea preservar su propia esfera de libertad y también la esfera de libertad ajena, entonces tiene que limitar 
Cuadernos Filosóficos. Segunda Época, XIII, 2016

su actividad eficiente sobre el mundo sensible y reducir su influencia sobre los demás a la actividad de exhortación. Por lo tanto, la exhortación es también la única actividad que le permite al otro conservar su esfera de libertad y alcanzar una conciencia de sí mismo. Quien se encuentra sometido a una fuerza extraña que no puede resistir, no puede tampoco ser consciente de sí mismo como un ser libre. Por ese motivo, Fichte afirma que mi propio acto de autoconciencia presupone que otro ser racional fuera de mí ha limitado su propia actividad, se ha mantenido dentro de los límites de su particular esfera de acción y sólo me ha dirigido una exhortación a actuar según mi propia capacidad de autodeterminación (Fichte, GA I/3, 350-351).

Ciertamente, este acto de exhortación puede comprenderse como un tipo de interacción comunicativa entre dos seres racionales. La exhortación es en sí misma una forma de comunicación, donde un ser racional dirige a otro una demanda, sin recurrir en ningún momento al uso de la fuerza. Bajo la influencia de esa exhortación, cada uno conserva su libertad y su capacidad de autodeterminación. Sobre la base de este concepto de exhortación, Fichte desarrolla su deducción trascendental del concepto de derecho, que presupone el mutuo reconocimiento de los seres racionales en el marco de una comunidad fundada en el principio de la legalidad jurídica. Por ese motivo, puede decirse que el reconocimiento entre seres racionales implica este tipo de acción comunicativa que Fichte denomina «exhortación». A través de la exhortación, cada sujeto conserva su propia esfera de acciones libres, sin interferir en la esfera del otro y sin avasallar sus derechos a través del uso irracional de la fuerza.

De este modo, Fichte incorpora a su teoría del derecho una profunda reflexión sobre el lenguaje y la intersubjetividad humana. No obstante, también en este caso resulta excesivo hablar de una anticipación del giro lingüístico por parte de Fichte. Por un lado, Fichte parece resistirse en todo momento a reconocer explícitamente que esta exhortación tiene la forma de un acto de habla. En verdad, la performatividad del lenguaje verbal no parece resultar imprescindible para que tenga efecto la exhortación. En su GNR de 1796/97, Fichte describe también la mera contemplación de la figura humana como una especie de exhortación silenciosa a reconocer al otro como un ser racional y a comportarse con él de acuerdo con la legalidad jurídica (Fichte, GA I/3, 376). Por otro lado, el concepto fichteano de exhortación tampoco presupone que el análisis del lenguaje constituya una instancia decisiva para la resolución de los problemas filosóficos. En definitiva, su deducción trascendental del concepto de derecho se mantiene siempre dentro de los límites del paradigma moderno de una filosofía de la conciencia, y nunca implica una reflexión sobre los elementos lingüísticos (sintácticos, semánticos o pragmáticos) de la propia argumentación filosófica. A pesar de sus diversos intentos de explicación o de justificación, el lenguaje sólo cumple una función secundaria 
Cuadernos Filosóficos. Segunda Época, XIII, 2016

en la filosofía trascendental de Fichte e incluso se considera como un obstáculo inevitable para la verdadera comprensión de las leyes de la razón.

Esta concepción del lenguaje como un medio necesario, pero al mismo tiempo imperfecto e insuficiente para expresar nuestros pensamientos, es desarrollada de manera precoz por Fichte en sus escritos Ueber den Unterschied des Geistes und des Buchstabens in der Philosophie de 1794. Como en otros escritos de la misma época, Fichte afirma que existe en el hombre un «impulso irresistible» (unwiderstehlicher Trieb) a buscar otro ser semejante fuera de sí. El hombre queda privado del verdadero goce del espíritu, cuando no encuentra alguien fuera de él con el cual poder comunicarse. Sin embargo, el único medio de comunicación posible entre el hombre y otro ser espiritual sólo puede ser el «mundo corpóreo» (Körperwelt). Un espíritu no puede actuar de manera inmediata sobre otro espíritu. Para poder ingresar con otro en una relación de acción recíproca, el espíritu tiene que producir fuera de sí una «manifestación» (Erscheinung), que exprese su idea espiritual, en la medida en que algo corporal puede expresar al espíritu. En este sentido, puede afirmarse que el espíritu es expresado o representado por un cuerpo (es decir, por un signo lingüístico). Y esta «representación» (Darstellung) del cuerpo tiene validez como una expresión del espíritu, solamente para aquél que también posee espíritu. Para aquello que carece de espíritu y de vida, nada posee efectivamente vida o espíritu (GA II/3, 319).

Ahora bien, un espíritu tampoco puede tener ninguna intención de actuar sobre otro como si fuera una simple cosa, sino que, a través de la expresión, más bien quiere ofrecerle al otro la oportunidad de desarrollar a partir de su propia actividad la idea espiritual que reside en él. Su «obra» (Werk) es elaborada con el espíritu, pero no es el espíritu mismo. De ese modo, un texto es un conjunto de signos materiales y fijos, que sólo puede adquirir algún sentido por medio de la referencia a su autor, que es un espíritu viviente. Yo mismo, explica Fichte, me encuentro en este instante en la misma situación. Yo les muestro a ustedes sólo un producto de mi actividad espiritual, el cual considero que representa o expresa mis propias ideas, pero no puedo ofrecerles la idea misma. Yo sólo puedo ofrecerles algo corporal en lugar de mis ideas. Mis palabras, que ustedes leen o escuchan, son precisamente estas cosas corporales. En sí mismas, estas palabras no son más que un «sonido vacío» (leerer Schall) o una «vibración» $(S t o \beta)$ en el aire que nos rodea. Pero el significado o «sentido» (Sinn) que las palabras puedan tener para ustedes, no es algo que igualmente yo pueda ofrecerles de manera inmediata. Ustedes deben desarrollar por sí mismos la idea a partir del contacto con estas palabras (GA II/3, 320). De este modo, Fichte comprendía el lenguaje como una instancia inevitable en la comunicación humana, pero siempre sometida a la peligrosa opacidad del signo lingüístico. Aún cuando se trata de un medio imprescindible de interacción, el carácter eminentemente corporal del 
Cuadernos Filosóficos. Segunda Época, XIII, 2016

signo representa siempre una posible amenaza para el éxito de la auténtica comunicación entre seres racionales.

\section{Conclusiones}

A mi juicio, la teoría fichteana del lenguaje no consigue superar el esquema básico de la filosofía moderna de la conciencia, así como tampoco anticipa las futuras consecuencias del denominado giro lingüístico. A pesar de su esfuerzo de deducción trascendental, Fichte no atribuye demasiada importancia al lenguaje en el marco de su exposición sistemática de la WL durante el período de Jena (1794-1799), y sólo lo considera como un objeto posible de la investigación filosófica. Esta comprensión del lenguaje por parte de Fichte no se modifica substancialmente en sus escritos posteriores. Es cierto que su Rede an die deutsche Nation invierte la relación entre pensamiento y lenguaje que generalmente puede observarse en el contexto de una filosofía de la conciencia. En este escrito del período de Berlín (1800-1814), el pensamiento de los individuos parece estar determinado por el lenguaje de la comunidad y no a la inversa. Sin embargo, no resultaría legítimo sostener que Fichte anticipa de este modo los posteriores resultados del giro lingüístico de la filosofía. En realidad, Fichte nunca formula de manera consecuente una teoría explícita sobre las condiciones lingüísticas del conocimiento, de la argumentación filosófica o de las acciones humanas en general. Es decir, Fichte no deja de concebir el lenguaje como un posible objeto de conocimiento entre otros y nunca lo incorpora a la WL como una condición indispensable de la propia reflexión filosófica. En su filosofía, el lenguaje se convierte en objeto de una deducción trascendental, pero nunca en una condición de posibilidad del propio procedimiento deductivo. A pesar de los diversos intentos de explicación o de justificación, el lenguaje sólo cumple una función secundaria en la filosofía trascendental de Fichte e incluso se considera como un obstáculo inevitable para la verdadera comprensión de las leyes de la razón.

En este punto, se hace evidente un fuerte contraste con la concepción del lenguaje que prevalece dentro del actual panorama de la filosofía contemporánea. Después del giro lingüístico, la filosofía le atribuye una función decisiva al lenguaje y a la acción comunicativa. Desde la perspectiva pragmática de Karl-Otto Apel, por ejemplo, el lenguaje no sólo se ha convertido en un objeto tematizado por la reflexión filosófica, sino además en el medium donde se desarrolla esa misma reflexión. Es fácil advertir que la filosofía contemporánea ya no se ocupa esencialmente de la conciencia, sino del lenguaje como su objeto privilegiado de estudio. Sin embargo, esta circunstancia no la convierte simplemente en una filosofía que adopta el lenguaje como un objeto posible de conocimiento. La filosofía contemporánea del lenguaje cumple ahora la misma función que la 
Cuadernos Filosóficos. Segunda Época, XIII, 2016

moderna crítica del conocimiento. Esto significa, más específicamente, que la filosofía del lenguaje no es simplemente una tematización del lenguaje como un posible objeto de conocimiento entre otros, sino que ella misma se convierte en una reflexión sobre las condiciones lingüísticas de posibilidad del conocimiento. El problema de la pretensión de verdad ya no puede resolverse a partir de la evidencia o de la certeza de una conciencia individual, sino a partir de una formación intersubjetiva y argumentativa de consenso (Apel, 1999, pp. 311-312).

Esta posición es compartida por Kuhlmann, quien explica que el giro lingüístico transforma el análisis filosófico del lenguaje en el paradigma predominante para la filosofía del siglo XX. Antes del giro lingüístico, el lenguaje sólo funcionaba como un objeto de conocimiento. Luego del giro lingüístico, la filosofía empieza a comprender el lenguaje como un presupuesto decisivo del conocimiento. Esto significa básicamente que, a través del giro lingüístico, la filosofía del lenguaje se transforma en philosophia prima. De este modo, la pregunta por las condiciones de posibilidad del conocimiento se convierte en la pregunta por las condiciones de posibilidad de enunciados sobre el mundo válidos intersubjetivamente. La instancia última y decisiva para la resolución del problema filosófico sobre la validez del conocimiento ya no puede encontrarse en la evidencia subjetiva de una conciencia individual, sino en la formación argumentativa de consenso por parte de una comunidad de investigadores (Kuhlmann, 1985, pp. 15-16).

Evidentemente, Fichte no pudo anticipar las consecuencias del giro lingüístico, en el sentido que se acaba de mencionar. No obstante, considero que Fichte realiza una notable contribución a un debate filosófico de su época, que también tiene repercusiones en la actualidad: incorpora de forma explícita la perspectiva de la filosofía trascendental al debate sobre el origen y la validez del lenguaje humano. Después del giro lingüístico, esta perspectiva de la filosofía trascendental ha sido notablemente recuperada por la propuesta de Karl-Otto Apel y Wolfgang Kuhlmann. Gracias a esta recuperación, tampoco el debate contemporáneo sobre el lenguaje puede prescindir de la perspectiva trascendental. Aunque con considerables limitaciones, Fichte ya había realizado en su época una operación similar: extrajo el tema del lenguaje del ámbito contingente de la experiencia para situarlo en el horizonte trascendental de la reflexión filosófica. De este modo, la filosofía fichteana intentó evitar las consecuencias relativistas de una teoría empírica del lenguaje, que fuese elaborada únicamente a partir de datos falibles y contingentes. El proyecto filosófico de la modernidad (que pretendía fundamentar las decisiones y determinaciones del hombre en el uso de la razón) no quedaba así disuelto en una multiplicidad de reglas lingüísticas arbitrarias. De esta forma, tampoco quedaba eliminada la posibilidad de encontrar una fundamentación filosófica con validez universal y necesaria para todas las acciones humanas. Esa misma necesidad de encontrar un fundamento universalmente válido para el discurso y la praxis humana sigue representando un auténtico desafío para la actividad 
Cuadernos Filosóficos. Segunda Época, XIII, 2016

filosófica de nuestro tiempo. En ese sentido, la filosofía de Fichte aun puede ofrecer algunas contribuciones ciertamente estimulantes para el debate filosófico contemporáneo.

\section{Bibliografía}

Apel, K.A. (1999), Sprache als Thema und Medium der transzendentalen Reflexion. Zur Gegenwartssituation der Sprachphilosophie”. En Apel, K.A: Transformation der Philosophie, vol. II, Frankfurt am Main, Suhrkamp.

Dahlstrom, D.O. (2005), “The aesthetic holism of Hamann, Herder and Schiller”. En Ameriks, K. (ed.), The Cambridge Companion to German Idealism, New York, Cambridge University Press, 76-94.

Fichte, J.G. (1962 ss.), Gesamtausgabe der Bayerischen Akademie der Wissenschaften, Stuttgart/Bad Cannstatt: Frommann-Holzboog.

Hoffmann, T.S. (1997), "Die Grundlage der gesamten Wissenschaftslehre und das Problem der Sprache bei Fichte", en Fichte-Studien 10, 17-33.

Honneth, A. (2001): “Die transzendentale Notwendigkeit von Intersubektivität (Zweiter Lehrsatz: § 3)”. En: Merle, J-C. (ed.), Johann Gottlieb Fichte. Grundlage des Naturrechts, Berlin, Akademie Verlag, 63-80.

Kuhlmann, W. (1985), Reflexive Letztbegründung. Untersuchungen zur Transzendentalpragmatik, Freiburg-München, Verlag Karl Alber.

Siemek, M.J. (2003), "Fichtes und Hegels Konzept der Intersubjektivität”, en Fichte-Studien, 23, 5775.

Surber, J.P. (1996) Language and German Idealism: Fichte's Linguistic Philosophy, New Jersey. Humanity Press. 